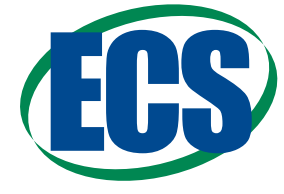

Jes Focus Issue on Mathematical Modeling of Electrochemical Systems at Multiple Scales in Honor of John Newman

\title{
Numerical Partitioning Model for the Koutecký-Levich Analysis of Electrochemical Flow Cells with a Combined Channel/Wall-Jet Geometry
}

\author{
S. A. Tschupp, ${ }^{\text {a,b }}$ S. E. Temmel, ${ }^{\text {a,c }}$ N. Poyatos Salguero, ${ }^{\text {a } J . ~ H e r r a n z, ~}{ }^{\mathrm{a}, *, \mathrm{z}}$ and T. J. Schmidt ${ }^{\mathrm{a}, \mathrm{d}, *}$ \\ ${ }^{a}$ Electrochemistry Laboratory, Paul Scherrer Institut, Villigen-PSI, 5232, Switzerland \\ ${ }^{b}$ Laboratory for Micro and Nanotechnology, Paul Scherrer Institut, Villigen-PSI, 5232, Switzerland \\ ${ }^{c}$ Materials Group, Paul Scherrer Institut, Villigen-PSI, 5232, Switzerland \\ ${ }^{d}$ Laboratory of Physical Chemistry, ETHZ, Zürich, 8093, Switzerland
}

\begin{abstract}
We present numerical studies of the flow profile and electrode currents for the hydrogen oxidation and oxygen reduction reactions in an electrochemical flow cell. Koutecký-Levich type equations are obtained by partitioning the electrode surface into flow profile regimes and their correlation to idealized wall-jet and channel electrode geometries. The precision of several Koutecký-Levich type equations is evaluated and it is shown that differences between the most commonly applied equations are negligible within the range of flow rates studied and are surpassed by experimental errors.

(c) The Author(s) 2017. Published by ECS. This is an open access article distributed under the terms of the Creative Commons Attribution 4.0 License (CC BY, http://creativecommons.org/licenses/by/4.0/), which permits unrestricted reuse of the work in any medium, provided the original work is properly cited. [DOI: 10.1149/2.0441711jes] All rights reserved.

(cc) BY
\end{abstract}

Manuscript submitted March 30, 2017; revised manuscript received May 30, 2017. Published June 21, 2017. This paper is part of the JES Focus Issue on Mathematical Modeling of Electrochemical Systems at Multiple Scales in Honor of John Newman.

Controlled experimental conditions are of prime importance in electroanalytical chemistry, be it battery, corrosion, electrocatalysis (e.g., reactions occurring in fuel cells or electrolyzers), and electrodeposition related research, respectively. Especially for the latter two, a defined transport of reacting species in the electrolyte to the electrode surface is crucial. In both fields, rotating electrodes such as the rotating disk electrode (RDE) and the rotating cylinder Hull cell $(\mathrm{RCH})$, respectively, have been the standard tool for electroanalytical investigations, due to their well-established mass transport conditions. Whereas the RCH exhibits a distinctively inhomogeneous current distribution similar to that of industrial electroplating baths, the RDE's uniformly accessible electrode surface enables the study of kinetic parameters and reaction pathways of multistep reactions such as the technologically relevant oxygen reduction and hydrogen oxidation reactions (ORR and HOR, respectively). Drawbacks of rotating electrodes, however, lie in the substrate geometry being limited to circular shapes, the need for bulk sample conductivity, and in the difficulties associated to their combination with online monitoring tools of great interest in electroanalytical chemistry. For such purposes, electrochemical flow cells based on different geometries have found widespread use in flow analysis due to their fast response time, high sensitivity and selectivity. ${ }^{1-4}$ Also in electrocatalysis, flow cells have been employed offering the possibility to work at elevated temperature and pressure, ${ }^{5-7}$ further enhanced by the coupling to spectroscopic tools. ${ }^{8-11}$ Other designs emphasize gas dosing by upstream mixing of two electrolytes, ${ }^{12,13}$ implementation of quartz crystal microgravimetry ${ }^{14,15}$ or the combination with in-situ X-ray absorption spectroscopy ${ }^{16-20}$ or scattering techniques. $^{20-23}$ Recently, a new class of electrochemical flow cells emerged from scanning electrochemical microscopes ${ }^{24,25}$ and scanning droplet cells, ${ }^{26-28}$ termed scanning flow cells (SFCs) or scanning droplet cell microscopes (SDCMs). ${ }^{29,30}$ A notable advantage is their ability to perform localized electrochemical measurements, thus greatly increasing throughput for the combinatorial screening of catalysts. Regardless of their wide range of application and advantages over RDEs, flow cells often involve complex flow velocity profiles and, thus, analytical solutions for the mass transport limited current as a function of flow rate are available only for generalized systems. ${ }^{31-36}$ While numerical methods can provide approximations for more complex cell designs, ${ }^{37-41}$ the commonly applied Koutecký-Levich (K-L) analysis to derive kinetic information from RDE experiments

\footnotetext{
*Electrochemical Society Member.

${ }^{\mathrm{z} E}$-mail: juan.herranz@psi.ch
}

is rendered unusable, due to the non-uniformity of the diffusion layer thickness and, thus, current density distribution across the electrode surface. ${ }^{31,42}$

With this motivation, we present an electrode partitioning method to obtain a numerical description of the current distribution at an electrochemical flow cell combining elements of wall-jet and channel flow profiles. We apply this method to an electrochemical flow cell used by our group ${ }^{43}$ and compare the K-L type equation obtained by numerical partitioning to the one commonly applied for the rotating disk electrode.

\section{Experimental Setup and Numerical Model}

Cell geometry.-A detailed description of the electrochemical flow cell and its comparison to the RDE has been published elsewhere. ${ }^{43}$ In brief, the cell consists of a copper plate into which the electrode is placed flush and an electrical contact is obtained via the top of the working electrode (WE) surface (see Figure 1). The WE compartment

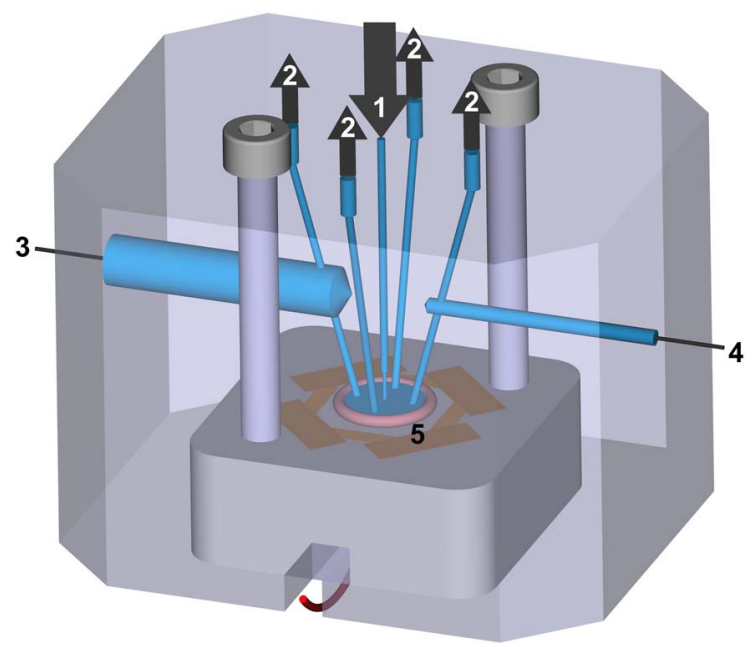

Figure 1. Sketch of the electrochemical flow cell. (1) Inflow from tube-in-tube (de)aeration system, (2) outlets, (3) RE compartment, (4) CE compartment, (5) WE compartment with O-ring sealing and top-contact to copper plate. Dimensions are not to scale for clarity of presentation. 
of the cell consists of a $0.5 \mathrm{~mm}$ diameter inlet nozzle and four $1 \mathrm{~mm}$ diameter outlet channels machined in a polyether ether ketone (PEEK) part placed $0.5 \mathrm{~mm}$ above the surface of the electrode embedded in a second PEEK part. The inlet is connected via high-performance liquid chromatography (HPLC) fittings to a syringe pump that supplies fresh electrolyte from an inert gas purged reservoir. Additionally, a tube-in-tube (de-) aeration system (based on devices applied in flow synthesis ${ }^{44-46}$ ) is connected upstream of the cell providing continuous, highly controllable gas dissolution into the electrolyte across a gas-permeable Teflon AF-2400 tubing, ${ }^{47}$ used for polarization experiments in different dissolved gas concentrations. Four outlets are arranged in a circular way around the inlet, two of which are intersected by compartments for reference and counter electrode (RE and $\mathrm{CE}$ ), respectively. The placement of both RE and CE downstream of the WE prevents the former two from acting as a source of contamination and eliminates the need for diffusion barriers. A Kalrez perfluoro elastomer O-ring sandwiched between WE and the upper PEEK part defines the cell volume and prevents the electrolyte from wetting the top-contact, thus eliminating it as a source of contamination. The distance between nozzle and electrode (i.e., the cell height) is fixed at $0.5 \mathrm{~mm}$ by spacers machined directly into the fluidic PEEK part of the cell, independent from O-ring compression. While several designs of the electrochemical flow cell suitable for different substrate sizes are in operation, the calculations presented herein are based on a design with a $9.25 \mathrm{~mm}$ O-ring inner diameter and a corresponding wetted area of approx. $100 \mathrm{~mm}^{2}$ typically used for $15 \times 15 \mathrm{~mm}^{2}$ substrates. Another cell geometry for $10 \times 10 \mathrm{~mm}^{2}$ samples is presented in the Supplementary Information.

Theoretical background.-The reciprocal total (i.e., measured) current $\left(1 / I_{t o t}\right)$ of an irreversible faradaic reaction of first order with respect to the reactant's concentration, as measured on a uniformly accessible electrode, can be described as a sum of the reciprocal of the kinetic current $\left(1 / I_{k i n}\right.$, i.e., the current that one would measure in the absence of mass transport limitations) and the reciprocal of the mass transport limited current $\left(1 / I_{\text {lim }}\right)$, according to the well-known Koutecký-Levich equation: ${ }^{42}$

$$
\frac{1}{I_{\text {tot }}}=\frac{1}{I_{\text {kin }}}+\frac{1}{I_{\text {lim }}}
$$

The only electrode to date fulfilling the need for uniform accessibility implied in the above equation is the rotating disk electrode (RDE). While Wakabayashi et al. applied Eq. 1 also to channel electrodes, ${ }^{5}$ its use for these has been scrutinized and, in agreement with experimental data, ${ }^{48}$ Scherson et al. later published a modified K-L type equation also for channel electrodes: ${ }^{49}$

$$
\frac{1}{I_{\text {tot }}}=\frac{1}{I_{\text {kin }}}+\frac{0.93}{I_{\text {lim }}}
$$

Similarly, a K-L type relation was proposed for wall-jet electrodes by Albery $^{50}$ and later applied for kinetic studies. ${ }^{51,52}$ If $I_{\text {tot }}<I_{\text {lim }} / 2$, the wall-jet electrode's accessibility is sufficiently uniform to obtain:

$$
\frac{1}{I_{\text {tot }}}=\frac{1.06}{I_{\text {kin }}}+\frac{1}{I_{\text {lim }}}
$$

Both modified K-L type relations (Eq. 2 and 3), however, have been derived for certain cell geometries, preventing their direct application to a generic electrochemical flow cell.

Governing equations.-The model was split into fluid dynamics and mass transport parts, each reflected by their governing equations and boundary layer properties. The fluid flow can be described by the incompressible Navier-Stokes equation under the assumption of constant temperature and electrolyte density throughout the cell: ${ }^{53}$

$$
\boldsymbol{\rho} \frac{\partial \mathbf{u}}{\partial t}=\mu \nabla^{2} \mathbf{u}-\nabla \mathbf{p}+\rho \mathbf{F}
$$

where $\rho$ is the density, $\mathbf{u}$ is the velocity tensor, $t$ is the time, $\mu$ is the fluid's dynamic viscosity, $\mathbf{p}$ is the pressure tensor and $\mathbf{F}$ is the
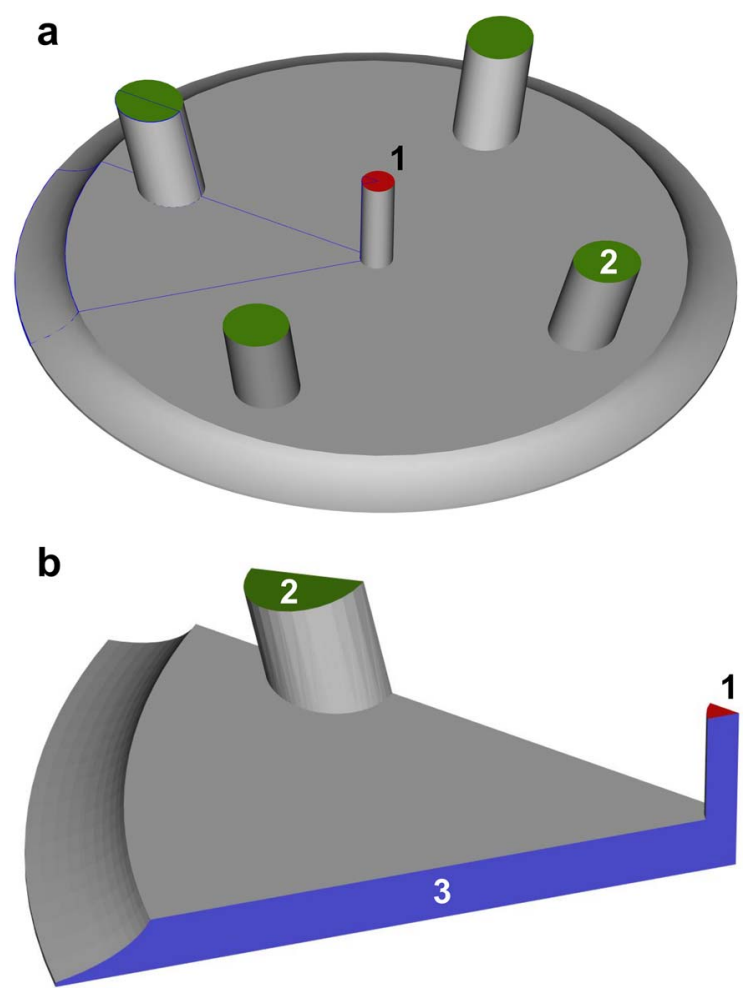

Figure 2. (a) Sketch of the complete model geometry and (b) actual domains used after symmetry operations. Inlet (1), outlet (2) and symmetry (3) boundaries are shown in red, green and blue, respectively.

external force tensor. Further, assuming a steady-state velocity profile $\left(\frac{\delta \mathbf{u}}{\delta t}=0\right)$ and negligible external force $(\mathbf{F}=0)$, Eq. 4 reduces to:

$$
\mu \nabla^{2} \mathbf{u}=\nabla \mathbf{p}
$$

The mass transport can be described by the Nernst-Planck equation. Using a supporting electrolyte providing sufficient conductivity and an abundance of spectator ions, the migration term can be neglected. The convective term takes advantage of the solution of the above fluid dynamics part, thus introducing the need for a coupling solver scheme: ${ }^{54}$

$$
\frac{\partial c}{\partial t}=\nabla \cdot(D \nabla c)-\mathbf{u} \cdot \nabla c+r
$$

where $c$ is the concentration of the reacting species, $D$ is its diffusion coefficient and $r$ describes an external chemical reaction rate. Under the assumption of a steady-state concentration profile $\left(\frac{\delta c}{\delta t}=0\right)$ as above and the absence of a chemical reaction rate term $(r=0)$, Eq. 6 reduces to:

$$
\nabla \cdot(D \nabla c)=\mathbf{u} \cdot \nabla c
$$

Boundary conditions. - The geometry and boundary conditions of the numerical simulation are summarized in Figure 2. To solve Eq. 5 for the fluid dynamics a linear flow velocity condition was assumed at the inlet (shown in red in Figure 2). For comparison with the measured polarization currents obtained using a syringe pump to drive the electrolyte in real experiments, ${ }^{43}$ the flow velocity was implemented as an average volumetric flow rate. For the outlets (shown in green in Figure 2), a pressure boundary condition was established with a value of $10^{5} \mathrm{~Pa}$, reflecting atmospheric pressure. Furthermore, the symmetrical design of the flow cell was exploited to reduce the computational domain to one-eighth of the original model by applying symmetry boundary conditions at the symmetry lines (shown in blue in Figure 2). All other boundary layers including the electrode were implemented as no-slip walls with a fluid velocity of zero relative to 
Table I. Model parameters for 5 wt.- $\% \mathrm{H}_{2} \mathrm{SO}_{4}(0.526 \mathrm{M})$

\begin{tabular}{|c|c|c|c|}
\hline Parameter & Symbol & Value (HOR) & Value (ORR) \\
\hline Temperature & $T$ & $293 \mathrm{~K}$ & $293 \mathrm{~K}$ \\
\hline Electrolyte density ${ }^{59}$ & $\rho$ & $1032 \mathrm{~kg} \mathrm{~m}^{-3}$ & $1032 \mathrm{~kg} \mathrm{~m}^{-3}$ \\
\hline Dynamic viscosity ${ }^{59}$ & $\mu$ & $1.112 \cdot 10^{-3} \mathrm{~Pa} \mathrm{~s}$ & $1.112 \cdot 10^{-3} \mathrm{~Pa} \mathrm{~s}$ \\
\hline Inlet concentration ${ }^{37,40}$ & $c_{\text {in }}$ & $1 \mathrm{~mol} \mathrm{~m}^{-3}$ & $1 \mathrm{~mol} \mathrm{~m}^{-3}$ \\
\hline Diffusion coefficient ${ }^{60}$ & $D$ & $4.58 \cdot 10^{-9} \mathrm{~m}^{2} \mathrm{~s}^{-1}$ & $2.01 \cdot 10^{-9} \mathrm{~m}^{2} \mathrm{~s}^{-1}$ \\
\hline Exchange current density ${ }^{56,57}$ & $i_{0}$ & $700 \mathrm{~A} \mathrm{~m}^{-2}$ & $2 \cdot 10^{-6} \mathrm{~A} \mathrm{~m}^{-2}$ \\
\hline Transfer coefficient ${ }^{57,61}$ & $\alpha$ & 0.5 & 1 \\
\hline Transferred electrons & $n$ & 2 & 4 \\
\hline Overpotential for diffusion control ${ }^{62,63}$ & $\eta$ & $0.5 \mathrm{~V}$ & $1 \mathrm{~V}$ \\
\hline
\end{tabular}

the wall. The initial velocity of the fluid body across the model was also set to zero.

For the mass transport problem introduced with Eq. 7, the inlet was described as an inflow boundary condition with an inlet concentration $c_{i n}$, whereas the outlet was implemented as an outflow boundary condition. Again, the symmetry lines were implemented as symmetry boundary conditions with a mass flux equal to zero in the direction normal to the boundary. To describe electrochemical reactions taking place at the electrode surface, a vanishing flux of reactive species was chosen as the boundary condition. For reversible electrochemical reactions (like the hydrogen oxidation/evolution reactions (HOR/HER) considered below) the flux $N$ can be described using the form of the Butler-Volmer equation below: ${ }^{55}$

$$
N=-\frac{i_{0}}{F n}\left[\frac{c_{f}^{\text {surface }}}{c_{f}^{\text {bulk }}} \exp \left(\frac{\alpha_{f} F}{R T} \eta\right)-\frac{c_{r}^{\text {surface }}}{c_{r}^{\text {bulk }}} \exp \left(-\frac{\alpha_{r} F}{R T} \eta\right)\right]
$$

where $i_{0}$ is the exchange current density, $F$ is the Faraday constant (96' $\left.485 \mathrm{C} \mathrm{mol}^{-1}\right), n$ is the number of transferred electrons per reacting molecule, and $c_{f}$ and $c_{r}$ are the concentrations of reacting species for the forward and the reverse reaction, respectively, at the electrode surface $\left(c^{\text {surface }}\right)$ and in the electrolyte bulk ( $c^{\text {bulk }}$, which is identical to $\left.c_{i n}\right)$. Additionally, $\alpha_{\mathrm{f}}$ and $\alpha_{\mathrm{r}}$ are the transfer coefficients for the forward and the reverse reaction, respectively, $\eta$ is the overpotential, $R$ is the ideal gas constant $\left(8.314 \mathrm{~J} \mathrm{~mol}^{-1} \mathrm{~K}^{-1}\right)$ and $T$ is the temperature. The roughness factor, customarily a part of this equation, has been assumed to be unity in this study, with the exchange current densities accounting for this. ${ }^{56,57}$

On the other hand, for irreversible reactions proceeding at high overpotentials (like the ORR, also regarded herein) Eq. 8 simplifies to:

$$
N=-\frac{i_{0}}{F n} \frac{\mathrm{c}_{\mathrm{f}}^{\text {surface }}}{\mathrm{c}_{f}^{\text {bulk }}} \exp \left(\frac{\alpha F}{\mathrm{RT}} \eta\right)
$$

In this work Eq. 8 was used to describe the HOR, assuming an equal concentration of protons at the electrode surface and in the bulk of the solution $\left(c_{r}^{\text {surface }} / c_{r}^{\text {bulk }}=1\right)$, while Eq. 9 was used without changes to model the ORR. All other boundary layers were implemented as no flux walls, for the mass transport problem being identical to the symmetry boundary condition. The initial concentration of the reacting species within the fluid body across the model was set to zero.

It should be noted that this numerical model does not hold for turbulent flow: while the Reynolds number $R e$ can be used to characterize the flow based on velocity, density, and viscosity of the electrolyte, the geometry's characteristic length changes along the fluid pathway, resulting in different values across the whole cell. However, within the nozzle where the characteristic length is at its smallest and the flow velocity largest, $R e$ is in the order of $20-100$, well below the transition to a turbulent flow regime at $R e \approx 2000$.

Meshing.-The model geometry was constructed with the computer-aided design (CAD) software CATIA v6 and transferred to the finite element method (FEM) software COMSOL Multiphysics 5.2 , where the geometry was refined and discretized within three re- gions. A "Free Triangular" mesh was applied to the electrode surface, whereas a "Free Tetrahedral" mesh was applied to the fluid body of the model. A "Boundary Layer" mesh was applied to all no-slip walls to increase the accuracy of the computation fluid dynamics calculations at the interface. At the interface between triangular and tetrahedral mesh close to the electrode surface, the number of boundary layer mesh elements was increased, thus increasing the resolution of the model in close vicinity of the electrode. In order to distinguish walljet and channel type electrode parts, the electrode surface was split into a center disk and a peripheral ring part, described by an areal ratio parameter $R_{A}=A_{\text {center }} /\left(A_{\text {center }}+A_{\text {periphery }}\right)$, corresponding to the disk-type fraction (i.e., $A_{\text {center }}$ ) of the whole electrode surface (i.e., $A_{\text {center }}+A_{\text {periphery }}$ ). Furthermore, a 2-step mesh refinement study was conducted, firstly decreasing the thickness of the first "Boundary Layer" mesh element by increasing the total amount of the latter until the local electrode current remained stable. ${ }^{58}$ Successively, the mesh element size for the "Free Triangular" mesh was decreased and chosen such that the total electrode current was steady upon a further change of the element size. Due to the partitioning mentioned above, the "Free Triangular" mesh was split into center and periphery parts as well and would change slightly upon variation of $\mathrm{R}_{\mathrm{A}}$. Therefore, the difference in total electrode current for $R_{A}=0.005$ and 0.25 was also assessed to assure a stable mesh. Details of the mesh refinement have been summarized in Table SI- 1 and the results thereof are presented in Figure SI-1 of the Supplementary Information.

\section{Results and Discussion}

In the following sections, the results of the computational simulations and a comparison of the model to experimental results will be presented. The reactions of choice were the HOR/HER and the ORR on platinum, both in acidic electrolyte. Kinetic and experimental parameters used for the numerical simulations were extracted from the literature and are summarized in Table I above.

Diffusion limited region.-In a first step, an overpotential $\eta$ sufficiently high to yield a concentration of the reacting species equal to zero at the electrode surface was chosen for Eqs. 8 and 9 (see overpotential for diffusion control in Table I). The resulting flow profiles - obtained by solving Eq. 5 - at a volumetric inlet flow rate of $2 \mathrm{ml} \mathrm{min}-1$ are shown in Figure 3a. Due to the small inlet nozzle diameter, the electrolyte enters the reaction compartment at high velocity, spreads over the electrode surface expanding and subsequently slows down. This behavior has been observed by Fuhrmann et al. for similar cell geometries. ${ }^{40,41}$ Solving Eq. 7 yields the total flux $N$ of reacting species flowing over the electrode surface, and the multiplication of $N$ by $F$ and $n$ results in the local current density $i_{l o c}$ shown in Figure $3 \mathrm{~b}$.

As expected from the velocity distribution, the highest concentration of reactive species is supplied to the center of the electrode with the smallest diffusion layer thickness and the highest local current density. Towards the outlets - shown as white circles in Figure $3 b$ the electrolyte velocity decreases, the supply of fresh reactants diminishes and the concentration boundary layer increases. Considering the 

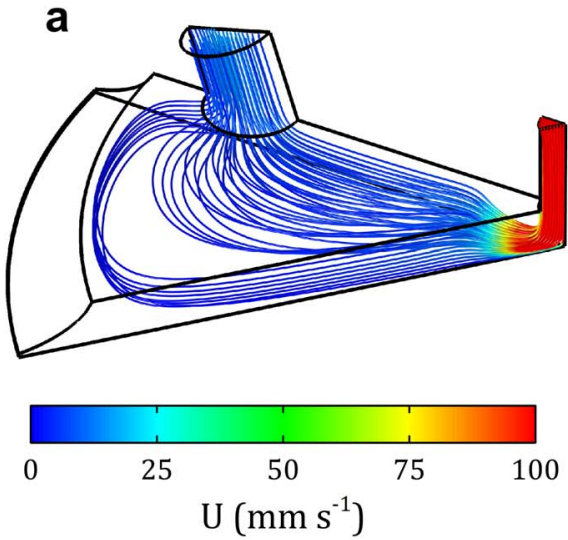



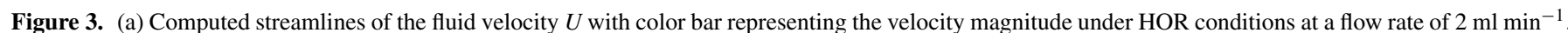

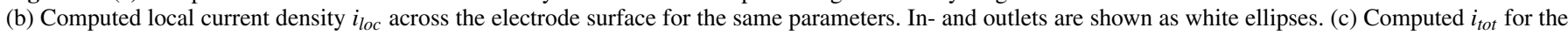
HOR at $\eta=0.5 \mathrm{~V}$ as function of $V_{\text {in }}$.

boundary layer as an array of finite resistors with a value proportional to the boundary layer thickness, the electrode current is highest in the electrode center and almost negligible in the region of stagnant electrolyte. This leads to a highly non-uniform current density distribution and highly localized probing of the electrode's surface. Measurements on not perfectly homogeneous electrodes are, therefore, difficult to interpret with the presented cell design and efforts have been directed at the optimization of the cell geometry via numerical modelling studies not shown herein.

Subsequently, integration of $i_{l o c}$ across the whole electrode surface yields the current density $i_{\text {tot }}$, corresponding to the measured current density in an experiment. The former is shown in Figure $3 \mathrm{c}$ as a function of the volumetric inlet flow rate for HOR conditions according to Table I. A power fit $i_{\text {tot }}=p \cdot V_{\text {in }}{ }^{q}$ in the range 0.5 to $5 \mathrm{ml} \mathrm{min}{ }^{-1}$ reveals an exponent $q$ of 0.40 , lying in-between the values of 0.33 and 0.5 that would be expected for a channel or a thin layer flow cell, respectively. ${ }^{32,34}$ Therefore, the application of either Eq. 2 or Eq. 3 to obtain a pure kinetic current $I_{\text {kin }}$ does not appear appropriate for the cell geometry considered herein. On the other hand, upon closer examination of the flow profile in the plane of the in- and outlet crosssection, one can distinguish two different flow regions depending on inlet flow rate (cf. Figure 4a). A layered flow profile indicative of laminar flow is observed in the region far from the inlet nozzle, whereas a wall-jet-like flow profile forms at the inlet and extends into the chan-

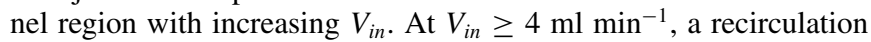
region becomes apparent at the inlet nozzle due to the constriction of an ideal wall-jet flow profile in the present case. In Figure 4b, the concentration of the reactive species is plotted normalized to the inlet concentration $\left(c_{i n}\right)$; it is apparent that, under these flow conditions, the diffusion boundary layer thickness is sufficiently small to not interfere with the height of the cell.

Electrode partitioning.-Subsequently, the electrode surface was partitioned in a center ("wall-jet") and a periphery ("channel") part, described by the areal ratio parameter $R_{A}=$ $A_{\text {center }} /\left(A_{\text {center }}+A_{\text {periphery }}\right)$ mentioned above. Calculating $i_{\text {tot }}$ for


Figure 4. Computed flow velocity profile (a) in the center plane at different volumetric inlet flow rates $V_{i n}$. Contour lines for comparison drawn at $U=40,80$, 120 , and $160 \mathrm{~mm} \mathrm{~s}^{-1}$, respectively. (b) The corresponding concentration plots under the same conditions. 


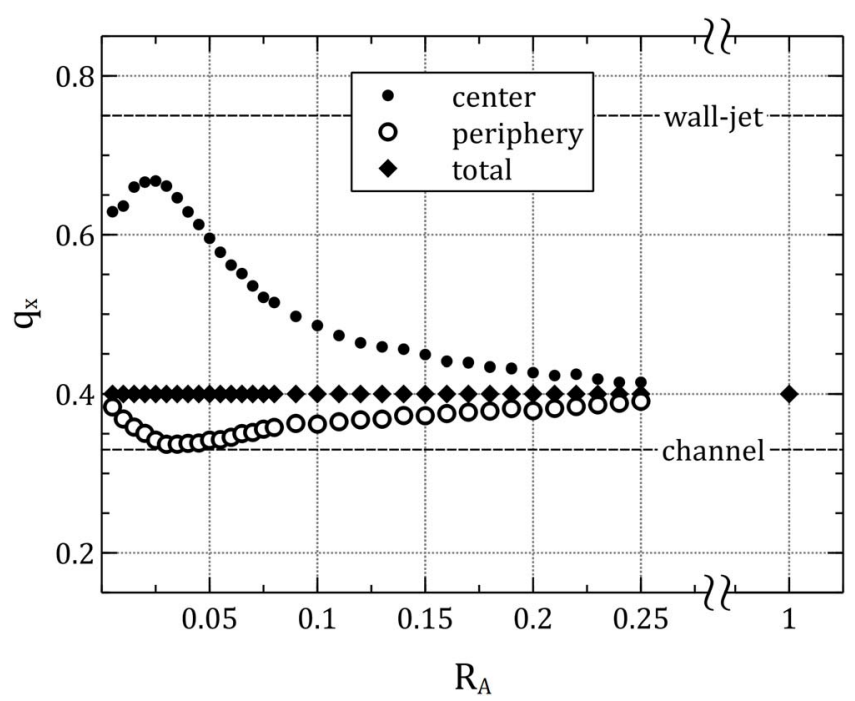

Figure 5. Computed exponent $q$ for center (solid circle marks) and periphery (hollow circle marks) parts of the electrode surface as a function of the areal ratio parameter of the electrode.

both center and periphery parts for volumetric inlet flow rates of 0.5-5 ml min ${ }^{-1}$, separate $i_{\text {tot,center }}$ and $i_{\text {tot,periphery }}$ values were obtained. Reproducing the approach followed above, the exponents $q_{\text {center }}$ and $q_{\text {periphery }}$ of the power fit $i_{\text {tot }}=p \cdot V_{i n}{ }^{q}$ were obtained for each ratio $R_{A}$ using a least squares method, and are displayed in Figure 5. The summation of $i_{\text {tot,center }}$ and $i_{\text {tot,periphery }}$ yields $i_{\text {tot }}$ for the whole electrode area as function of $R_{A}$. The exponent $q$ thereof was employed to verify the stability of the mesh for every partitioning step by comparison with the expected value for the non-partitioned model of 0.40 .

From Figure 5 it is apparent that an areal ratio of $R_{A}=0.025$ (corresponding to a disk radius of $860 \mu \mathrm{m}$ ) results in an excellent match between $q_{\text {periphery }}=0.34$ and the expected value of 0.33 for a channel electrode. ${ }^{32}$ At the same $R_{A}, q_{\text {center }}=0.67$, which is smaller than the value of 0.75 expected for an ideal wall-jet electrode, ${ }^{33}$ possibly due to the inability of a pure wall-jet flow profile to be formed due to the small distance between electrode and nozzle discussed above. The decrease of $q_{\text {center }}$ for $R_{A}<0.025$ can be explained considering that, approaching a center diameter in the order of the inlet nozzle's diameter, a wall-tube electrode arrangement is effectively formed. The flow profile thereof has been studied by several authors ${ }^{35,64,65}$ and a correlation of $I_{\text {lim }}$ to $V_{\text {in }}{ }^{1 / 2}$ has been proposed.

In a first approximation, the velocity profile at $R_{A}=0.025$ can therefore be interpreted as a superposition of wall-jet and channel flow profiles. Considering $I_{k i n}$ in the mass transport limited region to be infinite and inserting the corresponding equations for $I_{\text {lim }}$, the inverse of the total current plotted against the inverse of the flow rate to the respective power (cf. Table I) is expected to yield a line with an intercept of zero. Therefore, a plot of the reciprocal current computed at $\eta=0.5 \mathrm{~V}$ for the HOR at the center and the periphery parts as functions of $1 / V_{i n}^{3 / 4}$ and $1 / V_{i n}^{1 / 3}$, respectively, can be used to verify the applicability of the partitioning method, as shown in Figure 6 for $R_{A}=0.025$.

For the electrode partitioned at $R_{A}=0.025$, the intercept of the linear fit for the peripheral part in Figure $6 \mathrm{~b}$ is close to zero, which would correspond to $I_{\text {kin }}$ approaching infinity for large values of $V_{\text {in }}$ according to Eq. 2 and the obtained current being truly diffusion limited. On the other hand, the corresponding intercept for the center part in Figure 6a does not reach zero within the fitted range, which is expected as the corresponding exponent $q_{\text {center }}=0.68$ (cf. Figure 5) is significantly below the value of 0.75 assumed for an ideal wall-jet flow profile. Nevertheless, a linear summation of Eq. 2 and 3, weighted by $R_{A}$ according to Figure 5, leads to the following equation separating



Figure 6. Computed K-L plots for separate (a) center and (b) periphery parts of the electrode partitioned at $R_{A}=0.025$ for the HOR according to Table I. The corresponding linear fits reveal intercepts of 0.52 and $-0.071 /\left(\mathrm{mA} \mathrm{cm}^{-2}\right)$ for the center and the periphery, respectively.

kinetic and mass transport determined currents:

$$
\frac{1}{I_{\text {tot }}}=R_{A} \cdot\left(\frac{1.06}{I_{\text {kin }}}+\frac{1}{I_{\text {lim }}}\right)+\left(1-R_{A}\right) \cdot\left(\frac{1}{I_{\text {kin }}}+\frac{0.93}{I_{\text {lim }}}\right)
$$

which, after simplification, yields:

$$
\frac{1}{I_{t o t}}=\frac{1+0.06 \cdot R_{A}}{I_{\text {kin }}}+\frac{0.93+0.07 \cdot R_{A}}{I_{\text {lim }}}
$$

The proposed equation can be validated in the limiting cases of $R_{A}$; for $R_{A}=0$, Eq. 11 yields Eq. 2 for the channel electrode and in the opposite case of $R_{A}=1$, Eq. 3 for the wall-jet electrode is obtained. The non-zero intercepts pointed out in Figure 6 introduce an error regarding the accuracy of Eq. 11, and its order of magnitude is discussed below. With $R_{A}$ being very small for the flow cell geometry studied herein, Eq. 11 resembles Eq. 2 for the channel electrode. These findings agree with the correlation of $i_{\text {tot }}$ to $V_{\text {in }}$ with the power of 0.40 , which is closer to the correlation expected for a channel rather than a wall-jet flow profile, which has also been shown in Figure 6. The better the accuracy of $R_{A}$, (i.e., the closer $q_{\text {center }}$ is to 0.75 and $q_{\text {periphery }}$ is to 0.33) the more accurate the application of Eq. 11, for which numerical studies on reactions with kinetic parameters according to Table I are presented in the next paragraph.

Koutecký-Levich analysis._A combined parametric sweep of the overpotential $\eta$ and the inlet flow rate $V_{i n}$ was performed to obtain the computed HOR polarization plots shown in Figure 7. The curves match the Nernstian diffusion current $i_{\text {diff }}$ plotted as solid gray lines, which is observed for reactions with extremely fast kinetics and can be calculated using the equation below: ${ }^{66}$

$$
i_{\text {diff }}=i_{\text {lim }}\left[1-\exp \left(-\frac{2 F}{R T} \eta_{\text {HOR }}\right)\right]
$$

It is well known that, due to these ultra-fast kinetics, the study of the HOR in acidic electrolytes on Pt-surfaces requires tools enabling extremely high mass transport rates such as the $\mathrm{H}_{2}$ pump, ${ }^{56,67,68}$ scanning electrochemical microscopy, ${ }^{69,70}$ or micro-electrodes,${ }^{71}$ with a recent review given in Reference 72 . Thus, while the model accurately represents the diffusion-controlled polarization curves expected for 


\section{E (V) vs RHE}

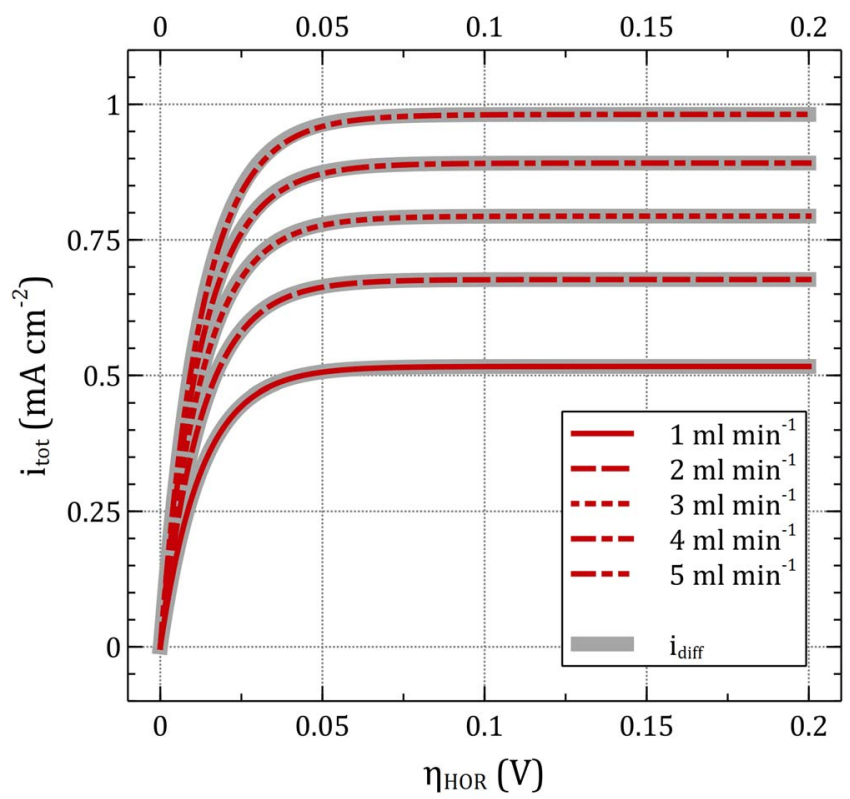

Figure 7. Computed HOR polarization curves obtained based on the parameters listed in Table I for different inlet flow rates. The corresponding diffusion currents $i_{\text {diff }}$ calculated according to Eq. 12 are shown as solid gray lines.

measurements carried out in a limited mass transport regime (such as the RDE), the impossibility to derive kinetic information from these computed polarization curves makes these unsuitable to demonstrate the application of Eq. 11 for a complete K-L analysis.

Instead, ORR polarization curves were modelled applying Eq. 9 at the electrode surface and using the corresponding parameters listed in Table I. Comparison of Figure 8a with literature studies of the ORR on platinum reveals a good match of the current onset ${ }^{73}$ at an overpotential of $\approx 0.27 \mathrm{~V}$ (corresponding to a measured potential of $\approx 0.95 \mathrm{~V}$ vs. RHE, based on an equilibrium potential of $1.23 \mathrm{~V}$ vs. RHE). However, the limiting currents are significantly lower upon comparison with RDE studies (e.g., $\approx 2 \mathrm{~mA} \mathrm{~cm}^{-2}$ at $5 \mathrm{~mL} \mathrm{~min}^{-1}$ in Figure $8 \mathrm{a}$, vs. $\approx 6 \mathrm{~mA} \mathrm{~cm}^{-2}$ in an RDE rotating at $1^{\prime} 600 \mathrm{rpm}^{73}$ ); a discrepancy that can be attributed to a weaker source of convection in the flow cell considered in this study.

From the polarization curves, Tafel plots were obtained for both the non-corrected currents and after application of Eq. 11, shown in Figures $8 \mathrm{~b}$ and $8 \mathrm{c}$, respectively. It is apparent that, at low overpotentials in which the total current is barely affected by mass transport limitations (i.e., $\eta_{\mathrm{ORR}} \leq 0.35 \mathrm{~V}$ ), the Tafel slope $b$ can be obtained with sufficient accuracy without applying a diffusion correction. To report catalyst ORR-activities, a similar approach is conventionally used, that is, by comparing the kinetic currents at potentials in the range of $\approx 0.85$ to $\approx 0.95 \mathrm{~V}$ vs. RHE, instead of deriving $i_{0}$ via extrapolation of $i_{\text {kin }}$ over several orders of magnitude. The overlapping polarization currents in the kinetically controlled region at potentials of $E>0.85 \mathrm{~V}$ vs. RHE for different inlet flow rates in Figure 8a and the Tafel plots in Figure $8 \mathrm{~b}$ to $8 \mathrm{~d}$ confirm that this approach would also be suitable for comparison of catalysts tested with the presented flow cell, validating our previous assumptions. ${ }^{43,74}$ To further verify this point, experimental data of the surface-specific ORR-activity of polycrystalline platinum ( $\mathrm{Pt}_{\text {poly }}$, circles) and platinum nanoparticles supported on a high surface area carbon ( $\mathrm{Pt}_{\mathrm{HSAC}}$, diamonds) at $0.9 \mathrm{~V}$ vs. $\mathrm{RHE}$ derived from RDE-measurements in perchloric acid electrolyte are also plotted in Figure $8 \mathrm{c}$ for comparison. ${ }^{73}$ It should be noted that, for the model, sulfuric acid was used regarding input values of $\mu$, $\rho, c_{i n}, D$ (cf. Table I), whereas the experimental data used for this comparison was obtained in perchloric acid. While it is well-known that (bi-)sulfate species partially block the active sites in platinum and lead to lower ORR activities in sulfuric vs. perchloric acid, ${ }^{63,75}$ this effect is not accounted for in the simple Butler-Volmer equation applied in the numerical model (cf. Eq. 9), rendering these computed results comparable to experimental data obtained in a non-adsorbing electrolyte such as the perchloric acid used in those studies. Furthermore, the kinetic parameters $\left(i_{0}, \alpha\right)$ in Table I were derived from fuel cell measurements using platinum nanoparticles supported on a high
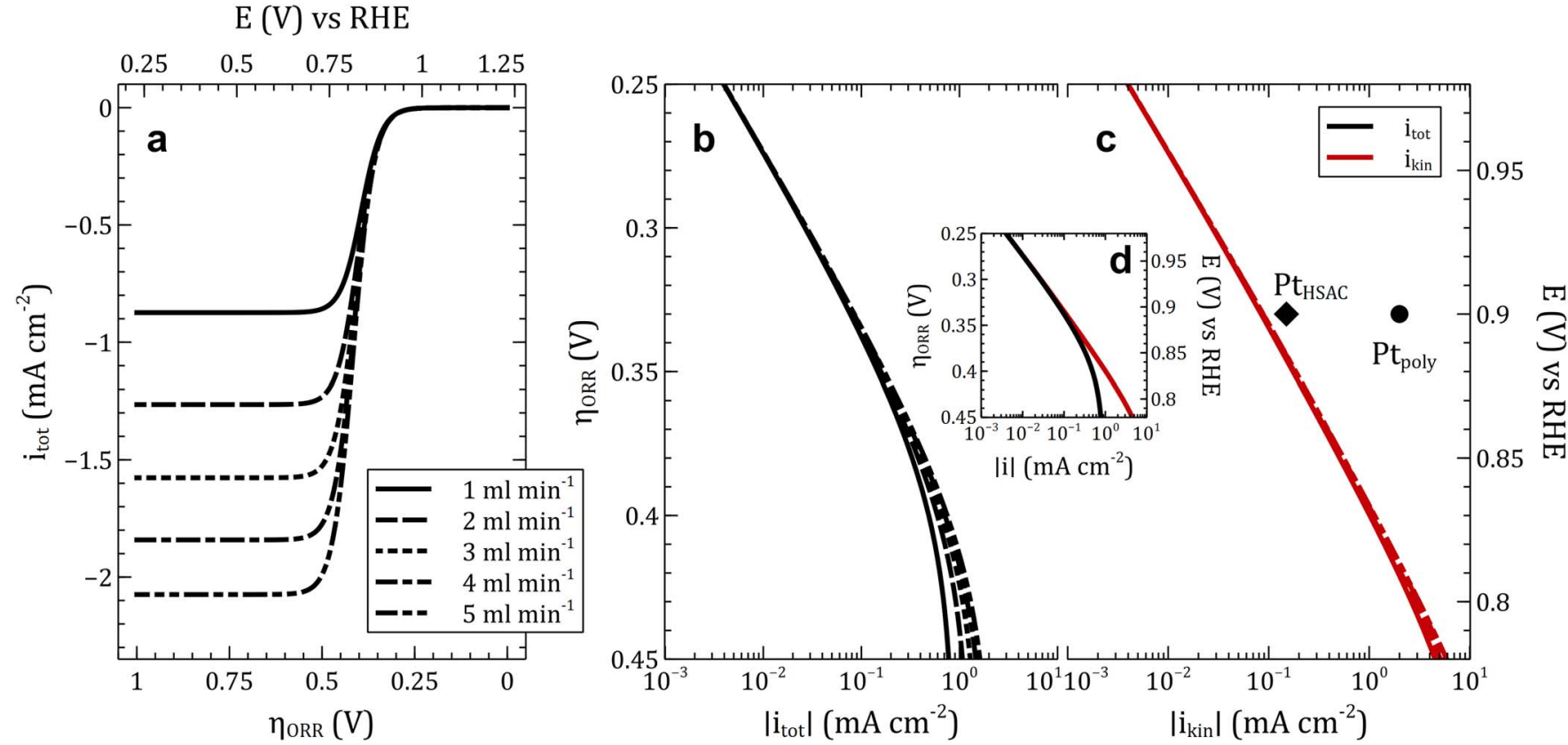

Figure 8. (a) Computed ORR polarization curves. (b) Corresponding Tafel plots for the non-corrected total currents and (c) kinetic currents after diffusion correction applying Eq. 11, and (d) in the same graph at a flow rate of $1 \mathrm{ml} \mathrm{min}^{-1}$ for ease of comparison. Experimental data at $0.9 \mathrm{~V}$ vs. RHE for platinum nanoparticles supported on a high surface area carbon $\left(\mathrm{Pt}_{\mathrm{HSAC}}\right)$ and polycrystalline platinum $\left(\mathrm{Pt}_{\mathrm{poly}}\right)$ from the literature ${ }^{73}$ are shown as black diamond and a black circle, respectively. 


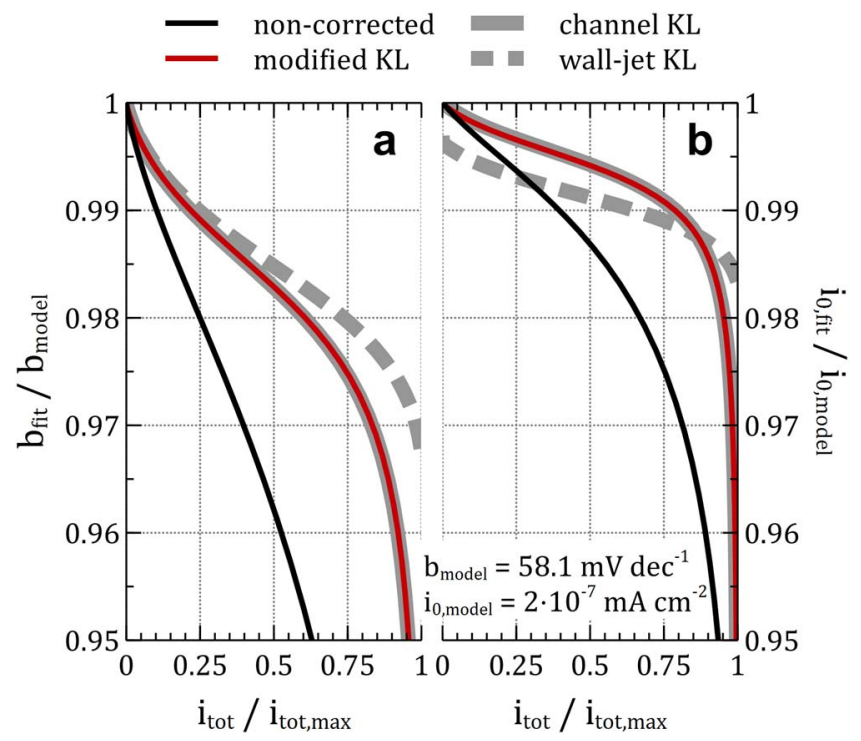

Figure 9. Computed precision of the K-L analysis at $V_{\text {in }}=2 \mathrm{ml} \mathrm{min}{ }^{-1}$ for (a) Tafel slope $b_{f i t}$ and (b) exchange current density $i_{0, f i t}$ compared to model input values $b_{\text {model }}$ and $i_{0, \text { model }}$, respectively, for different K-L type equations: non-corrected currents (black), Eq. 11 - electrode partitioning method (red), Eq. 2 - channel electrode (solid gray), Eq. 3 - wall-jet electrode (dashed gray).

surface area carbon as the cathode catalyst ${ }^{57}$ instead of polycrystalline platinum due to the lack of reliable exchange current densities thereof, which explains the excellent match of the computed $i_{k i n}$ value at $0.9 \mathrm{~V}$ vs. RHE with the corresponding experimental data for $\mathrm{Pt}_{\mathrm{HSAC}}$. This discrepancy between the specific activities for Pt-based catalysts with different dispersions/surface areas has been observed before and correlated to the ECSA in an experimental master curve, ${ }^{76,77}$ as well as supported by earlier DFT calculations. ${ }^{78}$

If, as in this study, a complete K-L analysis is to be considered, the application of Eq. 11 extends the range in which a linear fit yields $b$ and $i_{0}$, increasing its reliability. However, since K-L type equations are not applicable within the region of complete mass transport limitation (i.e., at $\eta>0.6 \mathrm{~V}$ ) due to insufficient current resolution, it is interesting to assess the maximum value of $I_{\text {tot }} / I_{\text {tot,max }}$ at which a Tafel fit yields $b$ and $i_{0}$ with sufficient accuracy. In Figures $9 \mathrm{a}$ and $9 \mathrm{~b}$ these values are shown as solid red lines after diffusion correction with Eq. $11 ; b$ and $i_{0}$ as obtained from the non-corrected (mass transport limited) currents are shown as black lines. For comparison, the currents were also corrected applying the equations for channel and wall-jet electrodes (cf. Eqs. 2 and 3), which appear plotted as solid and dash thick gray lines, respectively. As expected from the similarity of Eqs. 11 and 2, the accuracy of the respective Tafel fits matches as well. Following diffusion correction with Eq. 3, though, the Tafel fit yields a less accurate $i_{0}$ (Figure $9 \mathrm{~b}$ ) even for low ratios of $I_{\text {tot }} / I_{\text {tot,max }}$; however, $b$ (Figure 9a) is more accurate. It should be noted that, contrary to the common experimental practice referred to above,${ }^{66}$ the extension of the fitting region to $I_{\text {tot }} / I_{\text {tot, max }}$ values $>0.5$ still yields $b$ and $i_{0}$ with high accuracy. This can in part be attributed to the fact that the computed currents at low overpotentials are therefore not affected by mass transport losses and are much more accurate in the numerical simulation as compared to experimental studies. Nevertheless, the precision at $I_{\text {tot }} / I_{\text {tot,max }}>0.5$ is adversely affected by $V_{\text {in }}$, which has been chosen as $2 \mathrm{ml} \mathrm{min}^{-1}$ for the results presented in Figure 9 and the results need to be scrutinized in view of the application to experimental data.

Comparison to the rotating disk electrode.-Previous experimental and modelling studies on channel flow cells and SFCs found that the application of Eq. 1 for the rotating disk electrode results in sufficiently accurate diffusion correction for the K-L analysis of the ORR,



Figure 10. Computed precision of the K-L analysis at $V_{i n}=2 \mathrm{ml} \mathrm{min}^{-1}$ for (a) Tafel slope $b_{f i t}$ and (b) exchange current density $i_{0, f i t}$ compared to model input values $b_{\text {model }}$ and $i_{0 \text {,model }}$, respectively, for different K-L type equations: Eq. 11 - electrode partitioning method (red), Eq. 1 - rotating disk (black).

regardless of cell geometry. While Wakabayashi et al. did not present any explanation for applying Eq. 1 to their channel flow double electrode cell, ${ }^{5}$ Kulyk et al. performed numerical simulations similar to those in this work that demonstrated the applicability of the general $\mathrm{K}-\mathrm{L}$ equation with a $\pm 6 \%$ precision across the complete current range. ${ }^{37}$ However, the authors did not provide an analogous study for the K-L type equation of a channel electrode, which, considering the geometry of their SFC, would be a more reasonable choice. Applying Eq. 1 and assessing the accuracy of $b$ and $i_{0}$, as shown in Figures 10a and 10b, these observations are confirmed to extend to the geometry modelled in this study. In fact, a comparison with the kinetic parameters obtained after diffusion correction with Eq. 11 shows that the K-L equation for the rotating disk yields more accurate results than any of the other K-L type equations studied herein. Considering that the equations for the channel and the wall-jet electrode (Eqs. 2 and 3, respectively) are based on the general K-L equation and contain approximations implying numerical errors that were not discussed in the respective references, ${ }^{49,50}$ it is reasonable to assume that the linear combination of the aforementioned equations into Eq. 11 will result in error propagation. Regardless of this aspect, for the ensemble of cell geometry, range of flow rates, and kinetic parameters studied herein, we propose that the application of Eq. 1 is sufficient for the K-L analysis, for which errors are generally dominated by experimental factors.

\section{Conclusions and Outlook}

Fluid profile and mass transport in an electrochemical flow cell were studied via numerical simulation. A simple model was developed to accurately calculate the electrode current of the ORR and HOR both in the mass transport limited regime and upon consideration of electrode kinetics. A novel method to obtain K-L type equations for electrochemical flow cells with superimposed flow profiles of channel and wall-jet electrodes was presented and verified by assessing the precision of $b$ and $i_{0}$ for the ORR on platinum in acidic electrolyte obtained by the K-L analysis. While the method can be applied to any kind of such cells, the partitioning ratio $R_{A}$, determining the weight of the channel and wall-jet electrode parts for a linear combination to obtain a K-L type equation, is expected to differ for different cell geometries. One such geometry with different O-ring diameter and outlet channel angle is presented in the Supplementary Information. Similarly, extending the range of calculated flow rates will modify the 
value of $R_{A}$ obtained by the electrode partitioning method. For EFCs of arbitrary geometry, the model could be applied to calculate the corresponding polarization curves and, by variation of the numerators of a K-L type equation and assessment of its precision (cf. Figures 9 and 10), a K-L type equation for any EFC geometry could be obtained. It was shown that, for commonly used experimental conditions, the ORR currents at $0.85-0.95 \mathrm{~V}$ vs. RHE are largely unaffected by mass transport, confirming earlier assumptions to compare the catalysts' specific activities without the need for a complete K-L analysis. ${ }^{43,79,74}$ Furthermore, the applicability of the well-known K-L equation for the RDE was confirmed for the electrochemical flow cell presented herein and compared, over a large current range, to the respective equations for the channel ${ }^{49}$ and wall-jet ${ }^{50}$ electrodes, as well as for their linear combination regarded herein (cf. Eq. 11). It was found that the general K-L type equation for the RDE produces the most accurate results, similar to results presented by Kulyk et al. ${ }^{37}$ We assume this behaviour to be related to error propagation from the K-L equations for the channel and wall-jet electrodes.

\section{Acknowledgments}

Kindly acknowledged is financial support from Kabelwerke Brugg AG, Fachhochschule Nordwestschweiz (FHNW) and the research commission of Paul Scherrer Institut within a CROSS proposal.

\section{References}

1. P. T. Kissinger, "Amperometric and coulometric detectors for high-performance liquid chromatography," Anal. Chem., 49, 447A (1977).

2. J. A. Cooper and R. G. Compton, "Channel Electrodes - A Review," Electroanalysis, 10, 141 (1998).

3. J. Wang, "Electrochemical detection for microscale analytical systems: a review," Talanta, 56, 223 (2002)

4. M. Trojanowicz, "Recent developments in electrochemical flow detections-A review. Part I. Flow analysis and capillary electrophoresis," Anal. Chim. Acta., 688, 8 (2011)

5. N. Wakabayashi, M. Takeichi, M. Itagaki, H. Uchida, and M. Watanabe, "Temperature-dependence of oxygen reduction activity at a platinum electrode in an acidic electrolyte solution investigated with a channel flow double electrode," $J$. Electroanal. Chem., 574, 339 (2005).

6. Z. Jusys and R. J. Behm, "DEMS Analysis of Small Organic Molecule Electrooxidation: A High-Temperature High-Pressure DEMS study," ECS Trans, 16, 1243 (2008).

7. C. Chatzichristodoulou, F. Allebrod, and M. Mogensen, "High temperature and pressure electrochemical test station," Rev. Sci. Instrum, 84, 54101 (2013).

8. Z. Jusys, H. Massong, and H. Baltruschat, "A New Approach for Simultaneous DEMS and EQCM: Electro-oxidation of Adsorbed CO on Pt and Pt-Ru," J. Electrochem. Soc., 146, 1093 (1999).

9. H. Baltruschat, "Differential electrochemical mass spectrometry," J. Am. Soc. Mass Spectrom., 15, 1693 (2004).

10. M. Heinen, Y. X. Chen, Z. Jusys, and R. J. Behm, "In situ ATR-FTIRS coupled with on-line DEMS under controlled mass transport conditions-A novel tool for electrocatalytic reaction studies," Electrochim. Acta., 52, 5634 (2007)

11. M. Nesselberger, S. J. Ashton, G. K. H. Wiberg, and M. Arenz, "Design, development, and demonstration of a fully LabVIEW controlled in situ electrochemical Fourier transform infrared setup combined with a wall-jet electrode to investigate the electrochemical interface of nanoparticulate electrocatalysts under reactio," Rev. Sci. Instrum., 84, 74103 (2013)

12. M. Bergelin and M. Wasberg, "The impinging jet flow method in interfacial electrochemistry: an application to bead-type electrodes," J. Electroanal. Chem., 449, 181 (1998).

13. U. Koponen, T. Peltonen, M. Bergelin, T. Mennola, M. Valkiainen, J. Kaskimies, and M. Wasberg, "The impinging jet flow cell - a novel method for the study of PEM fuel cell material," J. Power Sources., 86, 261 (2000).

14. Z. Jusys and G. Stalnionis, "Wall-jet electrochemical quartz crystal microgravimetry: oxidation of Co(II)-ethylenediamine complexes on copper electrode," Electrochim. Acta., 45, 3675 (2000).

15. G. Stalnionis, L. Tamašauskaitè-Tamašiūnaitè, V. Pautieniene, and Z. Jusys, "CO electrooxidation on a polycrystalline Pt electrode: A wall-jet EQCN study," J. Electroanal. Chem., 590, 198 (2006).

16. S. Maniguet, R. J. Mathew, and A. E. Russell, "EXAFS of Carbon Monoxide Oxidation on Supported Pt Fuel Cell Electrocatalysts," J. Phys. Chem. B., 104, 1998 (2000).

17. J. McBreen, "In Situ X-Ray Absorption Studies of a Pt-Ru Electrocatalyst," J. Electrochem. Soc., 142, 3399 (1995).

18. M. E. Herron, S. E. Doyle, S. Pizzini, K. J. Roberts, J. Robinson, G. Hards, and F. C. Walsh, "In situ studies of a dispersed platinum on carbon electrode using X-ray absorption spectroscopy," J. Electroanal. Chem., 324, 243 (1992).
19. A. E. Russell and A. Rose, "X-ray Absorption Spectroscopy of Low Temperature Fuel Cell Catalysts," Chem. Rev., 104, 4613 (2004).

20. T. Binninger, E. Fabbri, A. Patru, M. Garganourakis, J. Han, D. F. Abbott, O. Sereda R. Kötz, A. Menzel, M. Nachtegaal, and T. J. Schmidt, "Electrochemical Flow-Cell Setup for In Situ X-ray Investigations I. Cell for SAXS and XAS at Synchrotron Facilities," J. Electrochem. Soc., 163, H906 (2016).

21. J. Tillier, T. Binninger, M. Garganourakis, A. Patru, E. Fabbri, T. J. Schmidt, and O. Sereda, "Electrochemical Flow-Cell Setup for In Situ X-ray Investigations II. Cell for SAXS on a Multi-Purpose Laboratory Diffractometer," J. Electrochem. Soc., 163, H913 (2016).

22. H. -G. Haubold, X. H. Wang, H. Jungbluth, G. Goerigk, and W. Schilling, "In situ anomalous small-angle X-ray scattering and X-ray absorption near-edge structure investigation of catalyst structures and reactions," J. Mol. Struct., 383, 283 (1996).

23. A. Braun, M. Bärtsch, B. Schnyder, R. Kötz, O. Haas, H. -G. Haubold, and G. Goerigk, "X-ray scattering and adsorption studies of thermally oxidized glassy carbon," J. Non. Cryst. Solids., 260, 1 (1999).

24. A. J. Bard, F. -R. F. Fan, J. Kwak, and O. Lev, "Scanning electrochemical microscopy Introduction and principles," Anal. Chem., 61, 132 (1989)

25. S. Amemiya, A. J. Bard, F. -R. F. Fan, M. V. Mirkin, and P. R. Unwin, "Scanning Electrochemical Microscopy," Annu. Rev. Anal. Chem., 1, 95 (2008).

26. A. W. Hassel and M. M. Lohrengel, "The scanning droplet cell and its application to structured nanometer oxide films on aluminum," Electrochim. Acta., 42, 3327 (1997).

27. T. Suter and H. Böhni, "A new microelectrochemical method to study pit initiation on stainless steels," Electrochim. Acta., 42, 3275 (1997).

28. M. M. Lohrengel, A. Moehring, and M. Pilaski, "Capillary-based droplet cells: Limits and new aspects," Electrochim. Acta., 47, 137 (2001).

29. A. K. Schuppert, A. A. Topalov, I. Katsounaros, S. O. Klemm, and K. J. J. Mayrhofer, "A Scanning Flow Cell System for Fully Automated Screening of Electrocatalyst Materials," J. Electrochem. Soc., 159, F670 (2012)

30. J. P. Kollender, M. Voith, S. Schneiderbauer, A. I. Mardare, and A. W. Hassel, "Highly customisable scanning droplet cell microscopes using 3D-printing," J. Electroanal. Chem., 740, 53 (2015).

31. V. G. Levich, Physicochemical Hydrodynamics, Prentice-Hall, Englewood Cliffs, N.J., (1962)

32. W. J. Blaedel, C. L. Olson, and L. R. Sharma, "The Tubular Platinum Electrode.," Anal. Chem., 35, 2100 (1963)

33. J. Yamada and H. Matsuda, "Limiting diffusion currents in hydrodynamic voltammetry - III. Wall jet electrodes," J. Electroanal. Chem. Interfacial Electrochem, 44 189 (1973)

34. K. Brunt and C. H. P. Bruins, "Evaluation of the characteristics of the differential amperometric detector in combination with anion-exchange chromatography, using l-ascorbic acid as test compound," J. Chromatogr. A., 172, 37 (1979).

35. H. B. Hanekamp and H. J. Van Nieuwkerk, "Theoretical considerations on the performance of electrochemical flow-through detectors," Anal. Chim. Acta., 121, 13 (1980).

36. H. B. Hanekamp and H. G. de Jong, "Theoretical comparison of the performance of electrochemical flow-through detectors," Anal. Chim. Acta., 135, 351 (1982).

37. N. Kulyk, S. Cherevko, M. Auinger, C. Laska, and K. J. J. Mayrhofer, "Numerica Simulation of an Electrochemical Flow Cell with V-Shape Channel Geometry," $J$. Electrochem. Soc., 162, H860 (2015).

38. R. G. Compton, C. R. Greaves, and A. M. Waller, "A general computational method for mass-transport problems involving wall-jet electrodes and its application to simple electron-transfer, ECE and DISP1 reactions," J. Appl. Electrochem., 20, 575 (1990).

39. J. A. Alden, Computational Electrochemistry, Oxford University, 1998

40. J. Fuhrmann, H. Zhao, E. Holzbecher, H. Langmach, M. Chojak, R. Halseid, Z. Jusys, and J. Behm, "Experimental and numerical model study of the limiting current in a channel flow cell with a circular electrode," Phys. Chem. Chem. Phys., 10, 3784 (2008).

41. J. Fuhrmann, A. Linke, H. Langmach, and H. Baltruschat, "Numerical calculation of the limiting current for a cylindrical thin layer flow cell," Electrochim. Acta., 55, 430 (2009)

42. B. Levich, "The theory of concentration polarisation," Discuss. Faraday Soc., 1, 37 (1947).

43. S. E. Temmel, S. A. Tschupp, and T. J. Schmidt, "A highly flexible electrochemica flow cell designed for the use of model electrode materials on non-conventional substrates," Rev. Sci. Instrum, 87, 45115 (2016).

44. L. Yang and K. F. Jensen, "Mass Transport and Reactions in the Tube-in-Tube Reactor," Org. Process Res. Dev., 17, 927 (2013).

45. M. Brzozowski, M. O'Brien, S. V. Ley, and A. Polyzos, "Flow Chemistry: Intelligent Processing of Gas-Liquid Transformations Using a Tube-in-Tube Reactor," Acc. Chem. Res., 48, 349 (2015).

46. C. J. Mallia and I. R. Baxendale, "The Use of Gases in Flow Synthesis," Org. Process Res. Dev., 20, 327 (2016)

47. P. R. Resnick and W. H. Buck, Teflon AF Amorphous Fluoropolymers, in: J. Scheirs, (Ed.), Modern Fluoropolymers, John Wiley \& Sons, Inc., New York, (1997), 397.

48. G. R. Engelhardt, R. Biswas, Z. Ahmed, S. N. Lvov, and D. D. Macdonald, "The use of channel flow cells for electrochemical kinetic studies in high temperature aqueous solutions," Electrochim. Acta., 52, 4124 (2007).

49. D. A. Scherson, Y. V. Tolmachev, Z. Wang, J. Wang, and A. Palencsar, "Extensions of the Koutecky-Levich Equation to Channel Electrodes," Electrochem. Solid-State Lett., 11, F1 (2008).

50. W. J. Albery, "The current distribution on a wall-jet electrode," J. Electroanal. Chem. Interfacial Electrochem, 191, 1 (1985). 
51. A. A. Karyakin, E. E. Karyakina, and L. Gorton, "The electrocatalytic activity of Prussian blue in hydrogen peroxide reduction studied using a wall-jet electrode with continuous flow," J. Electroanal. Chem., 456, 97 (1998).

52. Z. Jusys, J. Kaiser, and R. J. Behm, "A novel dual thin-layer flow cell double-disk electrode design for kinetic studies on supported catalysts under controlled masstransport conditions," Electrochim. Acta., 49, 1297 (2004).

53. G. K. Batchelor, An Introduction to Fluid Dynamics, Cambridge University Press, Cambridge, (1967)

54. J. Newman and K. E. Thomas-Alyea, Electrochemical Systems, 3rd Edition, John Wiley \& Sons, Inc., New York, (2004).

55. A. J. Bard and L. R. Faulkner, Electrochemical Methods - Fundamentals and Applications, Second Edi, John Wiley \& Sons, Inc., New York, NY, (2001).

56. J. Durst, C. Simon, F. Hasché, and H. A. Gasteiger, "Hydrogen Oxidation and Evolution Reaction Kinetics on Carbon Supported Pt, Ir, Rh, and Pd Electrocatalysts in Acidic Media," J. Electrochem. Soc., 162, F190 (2014).

57. K. C. Neyerlin, W. Gu, J. Jorne, and H. A. Gasteiger, "Determination of Catalyst Unique Parameters for the Oxygen Reduction Reaction in a PEMFC," J. Electrochem. Soc., 153, A1955 (2006)

58. B. Nine, How to Implement a Mesh Refinement Study, COMSOL Blog, https://www.comsol.com/blogs/how-to-implement-mesh-refinement-study, accessed June 27, 2016.

59. W. M. Haynes, ed., Concentrative properties of aqueous solutions: density, refractive index, freezing point depression, and viscosity, in: CRC Handb. Chem. Phys., 96th ed., CRC Press, Boca Raton, FL, (2016).

60. W. M. Haynes, ed., Diffusion of Gases in Water, in: CRC Handb. Chem. Phys., 96th ed., CRC Press, Boca Raton, FL, (2016).

61. J. Zheng, Y. Yan, and B. Xu, "Correcting the Hydrogen Diffusion Limitation in Rotating Disk Electrode Measurements of Hydrogen Evolution Reaction Kinetics," J. Electrochem. Soc., 162, F1470 (2015).

62. H. A. Gasteiger, N. M. Marković, and P. N. Ross, "H2 and CO Electrooxidation on Well-Characterized Pt, Ru, and Pt-Ru. 1. Rotating Disk Electrode Studies of the Pure Gases Including Temperature Effects," J. Phys. Chem., 99, 8290 (1995).

63. N. M. Marković, H. A. Gasteiger, and P. N. Ross, "Oxygen Reduction on Platinum Low-Index Single-Crystal Surfaces in Sulfuric Acid Solution: Rotating Ring-Pt(hkl) Disk Studies," J. Phys. Chem., 99, 3411 (1995).

64. S. L. Marchiano and A. J. Arvía, "Ionic mass transfer on fixed disk and conical electrodes under streaming solutions-I. Theoretical approach," Electrochim. Acta., 12, 801 (1967)

65. H. Matsuda, "Zur Theorie der stationären Strom-Spannungs-Kurven von RedoxElektrodenreaktionen in hydrodynamischer Voltammetrie, "J. Electroanal. Chem. Interfacial Electrochem, 15, 325 (1967).
66. W. Sheng, H. A. Gasteiger, and Y. Shao-Horn, "Hydrogen Oxidation and Evolution Reaction Kinetics on Platinum: Acid vs. Alkaline Electrolytes," J. Electrochem. Soc., 157, B1529 (2010).

67. K. C. Neyerlin, W. Gu, J. Jorne, and H. A. Gasteiger, "Study of the Exchange Current Density for the Hydrogen Oxidation and Evolution Reactions," J. Electrochem. Soc., 154, B631 (2007).

68. J. Durst, A. Siebel, C. Simon, F. Hasché, J. Herranz, and H. A. Gasteiger, "New insights into the electrochemical hydrogen oxidation and evolution reaction mechanism," Energy Environ. Sci., 7, 2255 (2014).

69. J. Zhou, Y. Zu, and A. J. Bard, "Scanning electrochemical microscopy," J. Electroanal. Chem., 491, 22 (2000).

70. C. G. Zoski, "Scanning Electrochemical Microscopy: Investigation of Hydrogen Oxidation at Polycrystalline Noble Metal Electrodes," J. Phys. Chem. B., 107, 6401 (2003).

71. V. S. Bagotzky and N. V. Osetrova, "Investigations of hydrogen ionization on platinum with the help of micro-electrodes," J. Electroanal. Chem. Interfacial Electrochem, 43, 233 (1973).

72. J. Herranz, J. Durst, E. Fabbri, A. Patru, X. Cheng, A. A. Permyakova, and T. J. Schmidt, "Interfacial effects on the catalysis of the hydrogen evolution, oxygen evolution and CO2-reduction reactions for (co-)electrolyzer development," Nano Energy, 29, 4 (2016)

73. K. Shinozaki, J. W. Zack, S. Pylypenko, B. S. Pivovar, and S. S. Kocha, "Oxygen Reduction Reaction Measurements on Platinum Electrocatalysts Utilizing Rotating Disk Electrode Technique," J. Electrochem. Soc., 162, F1384 (2015).

74. S. E. Temmel, E. Fabbri, D. Pergolesi, T. Lippert, and T. J. Schmidt, "Investigating the Role of Strain toward the Oxygen Reduction Activity on Model Thin Film Pt Catalysts," ACS Catal, 6, 7566 (2016).

75. N. M. Marković, R. R. Adžić, B. D. Cahan, and E. B. Yeager, "Structural effects in electrocatalysis: oxygen reduction on platinum low index single-crystal surfaces in perchloric acid solutions," J. Electroanal. Chem., 377, 249 (1994).

76. A. Rabis, P. Rodriguez, and T. J. Schmidt, "Electrocatalysis for Polymer Electrolyte Fuel Cells: Recent Achievements and Future Challenges," ACS Catal, 2, 864 (2012).

77. E. Fabbri, S. Taylor, A. Rabis, P. Levecque, O. Conrad, R. Kötz, and T. J. Schmidt, "The effect of platinum nanoparticle distribution on oxygen electroreduction activity and selectivity," Chem. Cat. Chem., 6, 1410 (2014).

78. G. A. Tritsaris, J. Greeley, J. Rossmeisl, and J. K. Nørskov, "Atomic-Scale Modeling of Particle Size Effects for the Oxygen Reduction Reaction on Pt," Catal. Letters., 141, 909 (2011).

79. S. E. Temmel, E. Fabbri, D. Pergolesi, T. Lippert, and T. J. Schmidt, "Tuning the Surface Electrochemistry by Strained Epitaxial Pt Thin Film Model Electrodes Prepared by Pulsed Laser Deposition," Adv. Mater. Interfaces., 1600222 (2016). 